\title{
SHEDDING NEW LIGHT ON EXPLODING STARS: TERASCALE SIMULATIONS OF NEUTRINO-DRIVEN SUPERNOVAE AND THEIR NUCLEOSYNTHESIS
}

Final Technical Report for the Period July 15, 2001 - November 30, 2006

Wick Haxton

University of Washington

Work Performed Under Cooperative Agreement No. DE-FC02-01ER41187 


\section{Scope of Work from TSI proposal}

This project was focused on simulations of core-collapse supernovae on parallel platforms. The intent was to address a number of linked issues: the treatment of hydrodynamics and neutrino diffusion in two and three dimensions; the treatment of the underlying nuclear microphysics that governs neutrino transport and neutrino energy deposition; the understanding of the associated nucleosynthesis, including the r-process and neutrino process; the investigation of the consequences of new neutrino phenomena, such as oscillations; and the characterization of the neutrino signal that might be recorded in terrestrial detectors. This was a collaborative effort with Oak Ridge National Laboratory, State University of New York at Stony Brook, University of Illinois at Urbana-Champaign, University of California at San Diego, University of Tennessee at Knoxville, Florida Atlantic University, North Carolina State University, and Clemson. The collaborations tie together experts in hydrodynamics, nuclear physics, computer science, and neutrino physics.

The University of Washington contributions to this effort include the further development of techniques to solve the Bloch-Horowitz equation for effective interactions and operators; collaborative efforts on developing a parallel Lanczos code; investigating the nuclear and neutrino physics governing the r-process and neutrino physics; and exploring the effects of new neutrino physics on the explosion mechanism, nucleosynthesis, and terrestrial supernova neutrino detection.

\section{Neutrino Response Functions for Neutrino-Nucleus Interactions}

Under TSI, a set of Lanczos algorithm tools were developed for efficiently evaluating weak interaction response functions and weak rates:

(1) a nuclear shell model code based on a new code architecture that generates the sparse matrix as a set of completely dense, overlapping block matrices. This structure will be of great help in constructing efficient codes for parallel machines that optimize the node communications problem;

(2) a linear operator inversion method that efficiently constructs the needed inclusive nuclear response functions over the entire $(\mathrm{q}, \mathrm{w})$ plane [1]. The technique is based on the use of Lanczos moments to reconstruct the response function in a basis of harmonic oscillator Slater determinants;

(3) input two-nucleon and three-nucleon effective interactions constructed by the BlochHorowitz method [2-4]; and

(4) a representation of these interactions in a special form - called a next-to-next-to-leadingorder (NNLO) interaction because up to four derivatives are included - that minimizes the node memory needed to store the effective interaction. We have shown the NNLO interaction to be accurate to $20 \mathrm{keV}$ through the shell-model space [5].

\section{Neutrino Signals from Supernovae: Background Neutrinos}

The nuclear response function work is integral to understanding the role of neutrinos in transporting energy and lepton number/flavor in the supernova explosion. It is equally important in understanding the response of terrestrial detectors to the supernova neutrino flux that arrives at earth. An important component of the University of Washington work has been the calculation of the continuous neutrino flux that comes from all past supernovae. This background flux 
depends on the integrated history of core collapse, including estimates of massive star evolution rates, estimates of the mass cuts that define the fraction of these stars that produce supernova neutrinos, and the cosmological effects that distort the spectra through red shifts [6-8]. These flux estimates were combined with our estimates of detector sensitivities to determine the limits we can currently place on this background neutrino source.

\section{Neutrino Nucleosynthesis}

The University of Washington group has led much of the work associated with neutrino-driven nucleosynthesis, including the neutrino process and neutrino effects on the r-process. This work has supported numerical efforts under TSI to investigate the r-process [9-12], work that suggests that some neutron-rich r-process nuclei could be produced in a wider variety of explosive environments than previously envisioned. Rapidly expanding, proton-rich environments can yield p-process and r-process nuclei, with yields sensitively dependent on the entropy, the electron fraction, and the dynamic timescale. The supporting TSI numerical work depended in large part on the development of amulti-zone nucleosynthesis code that fully coupled nuclear burning and material mixing in an implicit fashion. The applied math team at ORNL collaborated on iterative techniques and preconditioners for the sparse matrices involved.

\section{Neutrino Mixing}

The TSI neutrino group, which included University of Washington, explored the consequences of flavor off-diagonal piece of the neutrino self-interaction potential on the simultaneous transformation [13] of neutrinos and antineutrinos over broad ranges of neutrino energy. We found that the expected conditions of neutrino flux near the core's surface provide the necessary conditions for this mixing despite the fact that neutrino mass-squared differences are measured to be small. The effects on the neutrino-neutrino potential in moving the flavor transformation physics much deeper into the star, where it can effect supernova dynamics and alter explosive nucleosynthesis, is potentially of great importance.

\section{Selected refereed publications}

[1] Haxton, W. C., Nollett, K. M., and Zurek, K. M. (2005), "Piecewise moments method:

Generalized Lanczos technique for nuclear response surfaces", Physical Review C 72, 065501.

[2] Haxton, W. C. and Luu, T. (2002), "Perturbative Effective Theory in an Oscillator Basis?", Physical Review Letters 89, 182503.

[3] Luu, T. C., Bogner, S., Haxton, W. C., and Navrátil, P. (2004), "Effective interactions for the three-body problem", Physical Review C 70, 014316.

[4] Haxton, W. C. (2007), "Harmonic-Oscillator-Based Effective Theory", Proceedings of the 3rd ANL/MSU/INT/JINA RIA Theory Workshop, T. Duguet et al. (eds.), World Scientific, Vol. 15 (2007)

[5] Haxton, W. C. (2008), "Form of the Effective Interactions in Harmonic-Oscillator-Based Effective Theory,” Physical Review C 77, 034005 (2008). 
[6] Lunardini, C. (2006), "The diffuse supernova neutrino flux, supernova rate and SN1987A", Astroparticle Physics, 26, 190.

[7] Lunardini, C. (2006), "Diffuse neutrino flux from supernovae: Upper limit on the electron neutrino component from the nonobservation of antineutrinos at SuperKamiokande", Physical Review D, 73, 083009.

[8] Lunardini, C. (2005), "Probing Neutrino Properties with Supernovae", Open Issues in Core Collapse Supernova Theory, 451.

[9] Heger, A., Kolbe, E., Haxton, W. C., Langanke, K., Martínez-Pinedo, G., and Woosley, S. E. (2005), "Neutrino nucleosynthesis", Physics Letters B, 606, 258.

[10] Haxton, W. C. (2006), "Nuclear Astrophysics", AIP Conf. Proc. 870: Intersections of Particle and Nuclear Physics: 9th Conference CIPAN2006, vol 870, 33.

[11] Haxton, W. C. (2004), "Supernova Neutrino-Nucleus Physics and the r-Process", The rProcess: The Astrophysical Origin of the Heavy Elements and Related Rare Isotope Accelerator Physics, 73.

[12] Haxton, W. C. (2003), "Nuclear Problems in Astrophysics", Proceedings of the International Schoolof Physics Erico Fermi: From Nuclei and their Constituents to Stars, 93.

[13] Haxton, W. C. and Holstein, B. R. (2004), "Neutrino physics: An update", American Journal of Physics, 72, 18. 